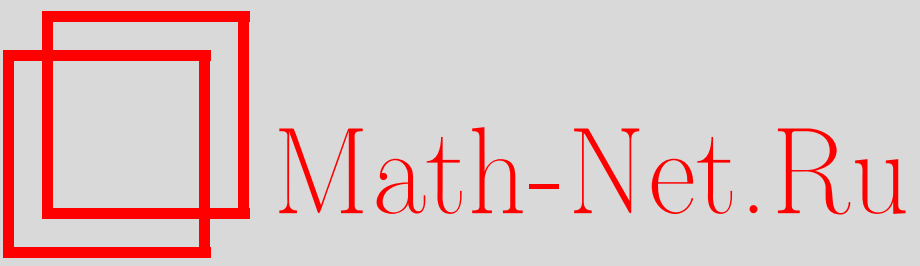

В. А. Андреев, В. И. Манько, О. В. Манько, Е. В. Щукин, Томография спиновых состояний, критерий перепутанности и неравенства Белла, ТМФ, 2006, том 146, номер 1, 172-185

DOI: https://doi.org/10.4213/tmf2017

Использование Общероссийского математического портала Math-Net.Ru подразумевает, что вы прочитали и согласны с пользовательским соглашением

http: //www . mathnet.ru/rus/agreement

Параметры загрузки:

IP : 54.162 .85 .209

26 апреля 2023 г., 12:50:48 
ТЕОРЕТИЧЕСКАЯ

И МАТЕМАТИЧЕСКАЯ

ФИЗИКА

Том 146, № 1

январь, 2006

(c) 2006 г. $\quad$ В. А. Андреев*, В. И. Манько*,

\author{
О. В. Манько*, Е. В. Щукин*
}

\title{
ТОМОГРАФИЯ СПИНОВЫХ СОСТОЯНИЙ, КРИТЕРИЙ ПЕРЕПУТАННОСТИ И НЕРАВЕНСТВА БЕЛЛА
}

\begin{abstract}
Дан обзор спиновой томографии квантовых состояний, в которой используются стандартные функции распределения вероятностей проекций спина на выделенные направления, содержащие ту же информацию о состояниях, что и матрица плотности. В рамках этого подхода показано, что справедливость неравенств Белла и их нарушения могут быть поняты как свойства совместных томографических функций распределения вероятностей двух спинов. Проведено сравнение результатов, полученных методами классической теории вероятностей, с результатами, получеными в рамках традиционной квантовой механики.
\end{abstract}

Ключевые слова: квантовая томография, вероятность, функции распределения, неравенство Белла.

\section{1. ВВЕДЕНИЕ}

Неравенства Белла [1] обычно используются, чтобы продемонстрировать отличие состояний в квантовой механике от состояний в классической механике. При этом для расчета средних и корреляций квантовых величин используется формализм матрицы плотности и квантовая процедура вычислений средних значений и корреляций. В классической механике используются стандартные формулы вычисления моментов, основанные на функции распределения вероятностей. Получающееся отличие (нарушение неравенств Белла) трактуется как квантовая нелокальность. При подобном анализе сравниваются результаты, полученные различными способами: на основе волновой функции или матрицы плотности и на основе стандартной теории вероятностей. Данная асимметрия в подходах связана с тем, что в квантовой механике считается невозможным задать состояние стандартной функцией распределения вероятностей, а концептуально необходимо использовать либо вектор состояния в гильбертовом пространстве (чистое состояние) либо матрицу плотности (смешанное состояние). Однако недавно было показано, что для непрерывных переменных типа координаты [2] и для дискретных переменных типа спина [3]-[6] квантовое состояние

*Физический институт им. П. Н. Лебедева РАН, Москва, Россия. E-mail: andrvlad@yandex.ru, manko@sci.lebedev.ru 
можно задавать обычными функциями распределения вероятностей, как в классической статистике. Данный подход называется вероятностным представлением квантовых состояний, или томографическим представлением. Как было показано в [7], вероятностное томографическое представление есть специфический вариант использования звездочного произведения, отличного от подхода Мойала [8], см. также [9]. Связь вероятностного представления с квантовой вероятностной мерой была найдена в [10]. Поскольку в вероятностном представлении квантовой механики квантовое состояние ассоциируется с обычными вероятностями, естественно использовать это представление для анализа неравенств Белла. В работе [11] были построены неравенства Белла для смешанных состояний, в явной форме учитывающие вероятности, с которыми чистые состояния входят в смешанное.

Основным объектом анализа в данной работе является совместная функция распределения вероятностей проекций спинов двух частиц на свои направления, которая полностью задает квантовое состояние. Зная эту совместную функцию распределения, можно востановить матрицу плотности состояния. Расчет средних значений, вторых и высших моментов для проекций спинов по стандартным формулам теории вероятностей полностью эквивалентен расчету этих величин по стандартным формулам квантовой механики с использованием матрицы плотности. Таким образом, задача анализа неравенств Белла с точки зрения квантово-механического формализма (в терминах матрицы плотности), сводится к изучению свойств совместных функций распределения вероятностей двух случайных величин в рамках стандартного формализма теории вероятностей.

Цель настоящей работы - показать, что нарушение неравенств Белла в квантовой механике для случая двух частиц со спинами $1 / 2$ может быть обнаружено путем анализа совместной функции распределения вероятностей, задающей квантовое состояние этих двух спинов, и найти в явном виде условия для совместных функций распределения вероятностей, при которых не происходит нарушения неравенств Белла. Второй задачей, решаемой в работе, является формулировка требований, разделяющих множество совместных функций распределения вероятностей на те, которые приводят к нарушению неравенств Белла и на те, которые им удовлетворяют, в рамках классической теории вероятностей, т.е. без обращения к квантовой механике. Мы покажем, что природа нарушений неравенств Белла, исследованных вначале для задач квантовой механики, на самом деле является чисто математической и связана с определенными различиями совместных функций распределения вероятностей. При этом могут быть использованы понятия томографической энтропии и томографической информации на унитарной группе, которые были введены в работе [12].

Вероятностные аспекты квантовой механики, включая и неравенства Белла, рассматривались в монографиях [13]. Анализу неравенств Белла с точки зрения теории вероятностей посвящены также работы [14]. В работах [15] показано, что квантовые корреляторы допускают классическое вероятностное представление, если учесть пространственную зависимость волновых функций, и если расстояние между детекторами достаточно велико. В работе [16] для некоторого частного класса двухча- 
стичных спиновых состояний неравенство Белла было переписано в терминах вероятностей измеряемых величин. В данной работе эта задача решается в наиболее общей постановке.

\section{2. СПИНОВАЯ ТОМОГРАФИЯ}

В квантовой механике наблюдаемые величины описываются операторами, действующими в гильбертовом пространстве состояний системы. В [7] в рамках вероятностного представления квантовой механики были решены задачи описания наблюдаемых величин функциями в фазовом пространстве системы и найдены формулы перехода от операторов к функциям, являющимся символами этих операторов, и обратного преобразования от функций к операторам. Этот подход является специфическим вариантом квантования, основанном на введении звездочного произведения функций, ассоциированных с физическими наблюдаемыми.

Рассмотрим случай спиновой томографии. Для случая одной частицы эта задача изучалась в работах [3]. В работах [4] было построено томографическое описание двухчастичного спинового состояния в случае, когда спины составляющих его одночастичных состояний проецируются на разные не связанные друг с другом оси координат. В работах [5] эта же задача была решена для случая, когда спины обеих частиц измеряются в одной системе кооординат. В работе [17] в терминах спиновой томографии был разобран парадокс Эйнштейна-Подольского-Розена.

Пусть оператор $\hat{A}^{(j)}$ для произвольных значений спина задается матрицей $A^{(j)}$ с элементами

$$
A_{m m^{\prime}}^{(j)}=\left\langle j m\left|\hat{A}^{(j)}\right| j m^{\prime}\right\rangle, \quad m=-j,-j+1, \ldots, j-1, j,
$$

где $|j m\rangle$ - это стандартные состояния с проекцией спина на ось $z$, равной $m$, т.е.

$$
\hat{j}_{z}|j m\rangle=m|j m\rangle \text {. }
$$

При этом

$$
\begin{gathered}
\hat{A}^{(j)}=\sum_{m=-j}^{j} \sum_{m^{\prime}=-j}^{j} A_{m m^{\prime}}^{(j)}|j m\rangle\left\langle j m^{\prime}\right|, \\
A_{m m}^{(j)}=w_{A}(m), \\
\sum_{m=-j}^{j} w_{A}(m)=\operatorname{Tr} \hat{A}^{(j)} .
\end{gathered}
$$

Рассмотрим диагональный элемент оператора $\hat{A}^{(j)}$ в другой системе отсчета:

$$
A_{m_{1} m_{1}}^{(j)}=\left(U \hat{A}^{(j)} U^{\dagger}\right)_{m_{1} m_{1}} .
$$

Унитарное преобразование поворота $U$ зависит от углов Эйлера $\alpha, \beta, \gamma$. Введем новое обозначение для матричных элементов оператора, переписав формулу (6) в 
виде

$$
\begin{aligned}
\widetilde{w}\left(m_{1}, \alpha, \beta, \gamma\right) & =\sum_{m_{1}^{\prime}=-j}^{j} \sum_{m_{2}^{\prime}=-j}^{j} D_{m_{1} m_{1}^{\prime}}^{(j)}(\alpha, \beta, \gamma) A_{m_{1}^{\prime} m_{2}^{\prime}}^{(j)} D_{m_{1} m_{2}^{\prime}}^{(j) *}(\alpha, \beta, \gamma)= \\
& =\operatorname{Tr}\left[A^{(j)} U^{\dagger}\left|m_{1}\right\rangle\left\langle m_{1}\right| U\right] .
\end{aligned}
$$

Введенная функция является томографическим символом оператора $\hat{A}^{(j)}$ и называется томограммой. Матричные элементы $D_{m_{1} m_{1}^{\prime}}^{(j)}(\alpha, \beta, \gamma)$ суть матричные элементы преобразования поворота,

$$
D_{m_{1} m_{1}^{\prime}}^{(j)}(\alpha, \beta, \gamma)=e^{i m_{1} \gamma} d_{m_{1} m_{1}^{\prime}}^{(j)}(\beta) e^{i m_{1}^{\prime} \alpha},
$$

где

$$
\begin{aligned}
d_{m_{1} m_{1}^{\prime}}^{(j)}(\beta)= & {\left[\frac{\left(j+m_{1}\right) !\left(j-m_{1}\right) !}{\left(j+m_{1}^{\prime}\right) !\left(j-m_{1}^{\prime}\right) !}\right]^{1 / 2} \times } \\
& \times\left(\cos \frac{\beta}{2}\right)^{m_{1}+m_{1}^{\prime}}\left(\sin \frac{\beta}{2}\right)^{m_{1}-m_{1}^{\prime}} P_{j-m_{1}}^{\left(m_{1}-m_{1}^{\prime}, m_{1}+m_{2}^{\prime}\right)}(\cos \beta),
\end{aligned}
$$

$P_{n}^{(a, b)}(x)$ - полином Якоби. Обратное к (7) преобразование, выражающее оператор через его символ, имеет вид

$$
\begin{aligned}
\sum_{j_{3}=0}^{2 j} \sum_{m_{3}=-j_{3}}^{j_{3}}\left(2 j_{3}+1\right)^{2} \sum_{m_{1}=-j}^{j} \int(-1)^{m_{1}} \widetilde{w}\left(m_{1}, \alpha, \beta\right) D_{0 m_{3}}^{\left(j_{3}\right)}(\alpha, \beta, \gamma) \times \\
\quad \times\left(\begin{array}{ccc}
j & j & j_{3} \\
m_{1} & -m_{1} & 0
\end{array}\right)\left(\begin{array}{ccc}
j & j & j_{3} \\
m_{1}^{\prime} & -m_{2}^{\prime} & m_{3}
\end{array}\right) \frac{d \omega}{8 \pi^{2}}=(-1)^{m_{2}^{\prime}} A_{m_{1}^{\prime} m_{2}^{\prime}}^{(j)}
\end{aligned}
$$

где

$$
\int d \omega=\int_{0}^{2 \pi} d \alpha \int_{0}^{\pi} \sin \beta d \beta \int_{0}^{2 \pi} d \gamma
$$

Полученные соотношения могут быть представлены в стандартной форме схемы квантования с помощью звездочного произведения [7]. Для этого введем неприводимый тензорный оператор группы $S U(2)$ :

$$
\widehat{T}_{L M}^{(j)}=\sum_{m_{1}, m_{2}=-j}^{j}(-1)^{j-m_{1}}\left\langle j m_{2} ; j-m_{1} \mid L M\right\rangle\left|j m_{2}\right\rangle\left\langle j m_{1}\right| .
$$

Операторы $\widehat{U}$ и $\widehat{D}$, задающие преобразование от оператора к его символу:

$$
\widetilde{w}\left(m_{1}, \alpha, \beta, \gamma\right)=\operatorname{Tr} \hat{A}^{(j)} \widehat{U}(m, \alpha, \beta, \gamma)
$$

и обратное преобразование от символа оператора к оператору (3):

$$
\hat{A}^{(j)}=\sum_{m_{1}=-j}^{j} \int d \omega \widetilde{w}\left(m_{1}, \alpha, \beta\right) \widehat{D}(m, \alpha, \beta, \gamma),
$$


могут быть представлены в виде

$$
\begin{aligned}
& \widehat{U}(m, \alpha, \beta, \gamma)=\sum_{L=0}^{2 j} \sum_{M=-L}^{L}(-1)^{j-m+M}\langle j m ; j-m \mid L 0\rangle D_{0-M}^{L}(\alpha, \beta, \gamma) \widehat{T}_{L M}^{(j)}, \\
& \widehat{D}(m, \alpha, \beta, \gamma)=\sum_{L=0}^{2 j} \sum_{M=-L}^{L}(-1)^{j-m+M} \frac{2 L+1}{8 \pi^{2}}\langle j m ; j-m \mid L 0\rangle D_{0-M}^{L}(\alpha, \beta, \gamma) \widehat{T}_{L M}^{(j)} .
\end{aligned}
$$

Как будет показано ниже, томограммы квантовых состояний являются полноправными распределениями вероятностей. Следовательно, в рамках томографического представления квантовой механики мы можем применить весь арсенал средств теории вероятностей к рассмотрению состояний квантово-механических систем, и это является преимуществом данного представления.

Рассмотрим в качестве примера произвольный оператор плотности, действующий в гильбертовом пространстве, который задает смешанное состояние в квантовой механике. Смешанное состояние описывается матрицей плотности $\rho$. В томографическом представлении квантовой механики состояние описывается томограммой, которая зависит от дополнительных параметров. Томограмма может быть как функцией непрерывных переменных (симплектическая томограмма), например, координаты, так и функцией дискретных переменных (спиновая томограмма, томограмма счета фотонов). Дополнительные параметры, от которых зависит томограмма, задают систему отсчета, в которой измеряется наблюдаемая. Замечательно, что существует взаимно однозначное соответствие, задаваемое обратимым отображением, между матрицей плотности $\rho$ и томограммой $\omega$ :

$$
\rho \leftrightarrow \omega
$$

В рамках спиновой томографии состояние частицы со спином $j$ и матрицей плотности $\rho$ описывается функцией распределения вероятности (спиновой томограммой $) \omega^{(j)}(m, \vec{n})$, где $m=-j,-j+1, \ldots, j$, а единичный вектор $\vec{n}=$ $(\sin \beta \cos \alpha, \sin \beta \sin \alpha, \cos \beta)$ задает точку на единичной сфере. Томограмма нормирована, т.е. для любого вектора $\vec{n}$ выполнено равенство

$$
\sum_{m=-j}^{j} \omega(m, \vec{n})=1
$$

Введем обозначение $D^{(j)}(u)$ для матрицы поворота. Тогда, по определению, томограмма связана с оператором плотности $\rho$ соотношением

$$
\omega(m, \vec{n})=\left\langle j m\left|D^{(j)+}(u) \rho D^{(j)}(u)\right| j m\right\rangle .
$$

Здесь $|j m\rangle$ - это стандартные состояния (2) с проекцией спина на ось $z$, равной $m$, а матрицы $D^{(j)}(u)$ - матрицы неприводимого представления группы $S U(2)$. Матрица $u(\alpha, \beta, \gamma)$ размера $2 \times 2$ является элементом этой группы,

$$
u(\alpha, \beta, \gamma)=\left(\begin{array}{cc}
\cos \left(\frac{\beta}{2}\right) e^{\frac{i}{2}(\alpha+\gamma)} & \sin \left(\frac{\beta}{2}\right) e^{-\frac{i}{2}(\alpha-\gamma)} \\
-\sin \left(\frac{\beta}{2}\right) e^{-\frac{i}{2}(\alpha-\gamma)} & \cos \left(\frac{\beta}{2}\right) e^{-\frac{i}{2}(\alpha+\gamma)}
\end{array}\right),
$$


а углы Эйлера $\alpha, \beta, \gamma$ - параметрами элемента $u$ группы $S U(2)$.

Из структуры формулы (17) видно, что томограмма не зависит от одного из углов Эйлера, а именно, от угла $\gamma$. Матрица плотности $\rho^{(j)}$ с матричными элементами $\rho_{m_{1} m_{2}^{\prime}}^{(j)}$ может быть восстановлена из томограммы с помощью следующего соотношения:

$$
\begin{aligned}
\sum_{j_{3}=0}^{2 j} \sum_{m_{3}=-j_{3}}^{j_{3}}\left(2 j_{3}+1\right)^{2} \sum_{m_{1}=-j}^{j} \int(-1)^{m_{1}} \widetilde{w}\left(m_{1}, \alpha, \beta\right) D_{0 m_{3}}^{\left(j_{3}\right)}(\alpha, \beta, \gamma) \times \\
\quad \times\left(\begin{array}{ccc}
j & j & j_{3} \\
m_{1} & -m_{1} & 0
\end{array}\right)\left(\begin{array}{ccc}
j & j & j_{3} \\
m_{1}^{\prime} & -m_{2}^{\prime} & m_{3}
\end{array}\right) \frac{d \omega}{8 \pi^{2}}=(-1)^{m_{2}^{\prime}} \rho_{m_{1}^{\prime} m_{2}^{\prime}}^{(j)},
\end{aligned}
$$

которое является аналогом равенства (10) для матрицы плотности. Томография кварковых состояний была предложена в работе [18], в работе [19] схема была обобщена и создана унитарная спиновая томография.

Рассмотрим две частицы со спинами $j_{1}$ и $j_{2}$. Базис в пространстве состояний системы может быть получен в виде произведения векторов

$$
\left|m_{1} m_{2}\right\rangle=\left|j_{1} m_{1}\right\rangle\left|j_{2} m_{2}\right\rangle .
$$

Матрица плотности $\rho$ двухчастичного спинового состояния системы связана со спиновой томограммой взаимно однозначным отображением [5]:

$$
\omega\left(m_{1}, m_{2}, \vec{n}_{1}, \vec{n}_{2}\right)=\left\langle m_{1} m_{2}\left|D^{\left(j_{1}\right)+}\left(u_{1}\right) \otimes D^{\left(j_{2}\right)+}\left(u_{2}\right) \rho D^{\left(j_{2}\right)}\left(u_{2}\right) \otimes D^{\left(j_{1}\right)}\left(u_{1}\right)\right| m_{1} m_{2}\right\rangle .
$$

Функция $\omega\left(m_{1}, m_{2}, \vec{n}_{1}, \vec{n}_{2}\right)$ задает совместное распределение вероятности двух дискретных случайных переменных $m_{1}$ и $m_{2}$, т.е. проекций спина на направления $\vec{n}_{1}=\left(\sin \beta_{1} \cos \alpha_{1}, \sin \beta_{1} \sin \alpha_{1}, \cos \beta_{1}\right)$ и $\vec{n}_{2}=\left(\sin \beta_{2} \cos \alpha_{2}, \sin \beta_{2} \sin \alpha_{2}, \cos \beta_{2}\right)$, соответственно. Функция $\omega\left(m_{1}, m_{2}, \vec{n}_{1}, \vec{n}_{2}\right)$ нормирована,

$$
\sum_{m_{1}=-j_{1}}^{j_{1}} \sum_{m_{2}=-j_{2}}^{j_{2}} \omega\left(m_{1}, m_{2}, \vec{n}_{1}, \vec{n}_{2}\right)=1 .
$$

Совместная томограмма зависит от точек на двух сферах, задающих единичные векторы $\vec{n}_{1}$ и $\vec{n}_{2}$. Томографическая вероятность одной частицы определяется через совместную томографическую вероятность двух частиц следуюшим образом:

$$
\omega\left(m_{1}, \vec{n}_{1}\right)=\sum_{m_{2}=-j_{2}}^{j_{2}} \omega\left(m_{1}, m_{2}, \vec{n}_{1}, \vec{n}_{2}\right) .
$$

\section{3. ПЕРЕПУТАННОСТЬ И РАЗЛИЧИМОСТЬ КВАНТОВЫХ СОСТОЯНИЙ}

Рассмотрим квантовые состояния двухмодовой системы с точки зрения их перепутанности или сепарабельности. Сепарабельными (неперепутанными) называются состояния, операторы плотности которых могут быть представлены в виде суммы 
прямых тензорных произведений операторов плотности подсистем с неотрицательными коэффициентами:

$$
\hat{\varrho}=\sum_{k} p_{k} \hat{\varrho}_{1}^{(k)} \otimes \hat{\varrho}_{2}^{(k)},
$$

причем коэффициенты удовлетворяют условию нормировки

$$
\sum_{k} p_{k}=1
$$

В рамках вероятностного представления квантовой механики мы можем сформулировать определение неперепутанности состояний на языке томограмм:

$$
w\left(m_{1}, m_{2}, \vec{n}_{1}, \vec{n}_{2}\right)=\sum_{k} p_{k} \bar{w}_{k}\left(m_{1}, \vec{n}_{1}\right) w_{k}\left(m_{2}, \vec{n}_{2}\right) .
$$

Обсудим критерий сепарабельности Переса-Городецкого [20], который основывается на следующих свойствах матрицы плотности:

$$
\operatorname{Tr} \varrho=1, \quad \varrho^{\dagger}=\varrho, \quad \varrho \geqslant 0
$$

Транспонированная матрица $R=\varrho^{\text {T }}$ также обладает всеми свойствами матрицы плотности:

$$
\operatorname{Tr} R=\operatorname{Tr} \varrho^{\mathrm{T}}=1, \quad R^{\dagger}=\left(\varrho^{\mathrm{T}}\right)^{\dagger}=R, \quad R=\varrho^{\mathrm{T}} \geqslant 0 .
$$

Неотрицательность матрицы, полученной транспонированием матрицы плотности, вытекает из неотрицательности исходной матрицы, поскольку собственные значения транспонированной матрицы совпадают с собственными значениями исходной матрицы. Так как матрица плотности является эрмитовой, то операция транспонирования эквивалентна операции комплексного сопряжения. Физический смысл операции комплексного сопряжения заключается в смене знака времени, т.е. в изменении направления течения времени в изучаемой системе на обратное.

Рассмотрим систему двух частиц со спинами $1 / 2$. Матрица плотности неперепутанных состояний двух частиц обладает следующими свойствами:

$$
\hat{\varrho}=\sum_{k} p_{k} \hat{\varrho}_{k}^{(1 / 2)} \otimes \varrho_{k}^{(1 / 2)}, \quad p_{k} \geqslant 0, \quad \sum_{k} p_{k}=1 .
$$

Если состояния являются перепутанными, то их матрица плотности не может быть представлена в виде суммы прямых тензорных произведений матриц плотности частиц с неотрицательными коэффициентами $p_{k}$, удовлетворяющими условию нормировки (25).

Рассмотрим транспонированную матрицу плотности неперепутанных квантовых состояний. Произведем операцию частичного транспонирования, т.е. транспонируем только матрицу плотности второй частицы:

$$
\hat{\varrho}=\sum_{k} p_{k} \hat{\varrho}_{k}^{(1 / 2)} \otimes\left(\hat{\varrho}_{k}^{(1 / 2)}\right)^{\mathrm{T}} .
$$


Физический смысл такой операции заключается в смене знака времени второй частицы. Матрицы $\left(\hat{\varrho}_{k}^{(1 / 2)}\right)^{\mathrm{T}}$ принадлежат к классу допустимых матриц плотности, так как они удовлетворяют всем необходимым свойствам.

Сформулируем критерий Переса-Городецкого. Матрицы плотности неперепутанных состояний при операции частичного транспонирования превращаются в допустимые матрицы плотности.

Рассмотрим в качестве примера состояние Вернера. Его матрица плотности имеет вид

$$
\varrho_{\mathrm{B}}=\left(\begin{array}{cccc}
\frac{1+p}{4} & 0 & 0 & \frac{p}{2} \\
0 & \frac{1-p}{4} & 0 & 0 \\
0 & 0 & \frac{1-p}{4} & 0 \\
\frac{p}{2} & 0 & 0 & \frac{1+p}{4}
\end{array}\right),
$$

где $p$ - действительный параметр.

Матричные элементы матрицы плотности, соответствующей оператору плотности $\hat{\varrho}$, вычисляются в стандартном базисе в пространстве состояний системы двух частиц со спинами, а именно, в базисе

$$
|1\rangle=|\uparrow\rangle|\uparrow\rangle, \quad|2\rangle=|\uparrow\rangle|\downarrow\rangle, \quad|3\rangle=|\downarrow\rangle|\uparrow\rangle, \quad|4\rangle=|\downarrow\rangle|\downarrow\rangle .
$$

Собственные значения матрицы плотности (27) состояния Вернера равны

$$
\lambda_{1}=\frac{1+3 p}{4}, \quad \lambda_{2}=\lambda_{3}=\lambda_{4}=\frac{1-p}{4} .
$$

Собственные значения (28) матрицы плотности состояния Вернера неотрицательны, если параметр $p$ удовлетворяет неравенству $-1 / 3 \leqslant p \leqslant 1$.

Произведем операцию частичного транспонирования матрицы плотности $\varrho_{\mathrm{B}}$, транспонировав матрицу плотности второй частицы. Получаем следующую матрицу:

$$
\varrho_{\mathrm{B}}^{p}=\left(\begin{array}{cccc}
\frac{1+p}{4} & 0 & 0 & 0 \\
0 & \frac{1-p}{4} & \frac{p}{2} & 0 \\
0 & \frac{p}{2} & \frac{1-p}{4} & 0 \\
0 & 0 & 0 & \frac{1+p}{4}
\end{array}\right),
$$

собственные значения которой равны

$$
\lambda_{1}=\frac{1-3 p}{4}, \quad \lambda_{2}=\lambda_{3}=\lambda_{4}=\frac{1+p}{4} .
$$

Собственные значения матрицы (29) неотрицательны, если $-1 \leqslant p \leqslant 1 / 3$. Следовательно, в соответствии с критерием Переса-Городецкого, если параметр $p$ удовлетворяет неравенству $-1 / 3 \leqslant p \leqslant 1 / 3$, то состояния Вернера являются сепарабельными. Если же $1 / 3<p \leqslant 1$, то состояние Вернера будет перепутанным, так как для данных значений параметра $p$ нарушено условие неотрицательности матрицы плотности $\varrho_{\mathrm{B}}^{p}(29)$. 


\section{4. НЕРАВЕНСТВА БЕЛЛА}

В вероятностном представлении квантовые состояния описываются томограммами, которые являются функциями распределения вероятности. В связи с этим рассмотрим неравенства Белла в вероятностном (томографическом) представлении квантовой механики и покажем, что для некоторых состояний неравенства Белла выполняются, а для некоторых состояний нарушаются. Рассмотрим плотность распределения вероятности $P(\lambda)$ произвольной случайной переменной $\lambda$,

$$
\int P(\lambda) d \lambda=1, \quad P(\lambda) \geqslant 0 .
$$

Введем случайные переменные $m_{1}(\lambda)$ и $m_{2}(\lambda)$, которые могут принимать значения 1 или -1. Тогда существует совместное распределение вероятностей $w\left(m_{1}, m_{2}\right)$ двух случайных переменных $m_{1}, m_{2}$, которое можно найти, зная явные выражения для функций $P(\lambda), m_{1}(\lambda), m_{2}(\lambda)$. Используя совместное распределение $w\left(m_{1}, m_{2}\right)$, можно вычислить все высшие моменты, в частности, корреляционный момент $\left\langle m_{1} m_{2}\right\rangle$ этого распределения. Кроме того, все моменты могут быть вычислены при помощи известной плотности распределения вероятности $P(\lambda)$ и функций $m_{1}(\lambda), m_{2}(\lambda)$. Корреляционный момент, являющийся средним значением от произведения двух переменных $m_{1}(\lambda)$ и $m_{2}(\lambda)$, имеет вид

$$
\left\langle m_{1} m_{2}\right\rangle=\int m_{1}(\lambda) m_{2}(\lambda) P(\lambda) d \lambda \leqslant 1 .
$$

Пусть переменные $m_{1}(\lambda), m_{2}(\lambda)$ зависят от дополнительных векторных параметров, задающих направление в пространстве. Введем векторы $\vec{a}, \vec{b}, \vec{d}, \vec{c}$, задающие четыре направления в пространстве. Получаем, что коррелятор переменной $m_{1}$, зависящий от направления $\vec{\alpha}=\vec{a}, \vec{d}$, и переменной $m_{2}$, зависящей от направления $\vec{\beta}=\vec{b}, \vec{c}$, имеет вид

$$
\left\langle M_{\alpha \beta}\right\rangle=\int m_{1}(\lambda)_{\alpha} m_{2}(\lambda)_{\beta} P(\lambda) d \lambda .
$$

Здесь и ниже индексы $\alpha, \beta$ означают, что величины, при которых они стоят, зависят от векторов $\vec{\alpha}, \vec{\beta}$. Исследуем следующее выражение:

$$
\begin{aligned}
\left\langle M_{a b}\right\rangle+ & \left\langle M_{a c}\right\rangle+\left\langle M_{d b}\right\rangle-\left\langle M_{d c}\right\rangle= \\
& =\int\left[m_{1}(\lambda)_{a}\left(m_{2}(\lambda)_{b}+m_{2}(\lambda)_{c}\right)+m_{1}(\lambda)_{d}\left(m_{2}(\lambda)_{b}-m_{2}(\lambda)_{c}\right)\right] P(\lambda) d \lambda .
\end{aligned}
$$

Очевидно, что

$$
\left|\left\langle M_{a b}\right\rangle+\left\langle M_{a c}\right\rangle+\left\langle M_{d b}\right\rangle-\left\langle M_{d c}\right\rangle\right| \leqslant 2 .
$$

Данное неравенство называется неравенством Белла.

Предположим, что совместное распределение случайных переменных $m_{1}, m_{2}$, принимающих значения \pm 1 , задается функцией $w\left(m_{1}, m_{2}, \vec{n}_{1}, \vec{n}_{2}\right)$, обладающей следующими свойствами:

$$
\sum_{m_{1}, m_{2}} w\left(m_{1}, m_{2}, \vec{n}_{1}, \vec{n}_{2}\right)=1, \quad w\left(m_{1}, m_{2}, \vec{n}_{1}, \vec{n}_{2}\right) \geqslant 0,
$$


и зависящей от дополнительных векторных параметров $\vec{n}_{1}, \vec{n}_{2}$, определяющих направления в пространстве. Рассмотрим корреляторы переменных $m_{1}, m_{2}$, связанных с теми же направлениями в пространстве, что и рассмотренные выше. Получаем

$$
\begin{array}{r}
\left\langle M_{\alpha \beta}\right\rangle=w \\
(1,1, \vec{\alpha}, \vec{\beta})-w(1,-1, \vec{\alpha}, \vec{\beta})-w(-1,1, \vec{\alpha}, \vec{\beta})+ \\
+w(-1,-1, \vec{\alpha}, \vec{\beta}), \quad \alpha=a, d, \quad \beta=b, c .
\end{array}
$$

Выполнение или нарушение неравенства Белла (38) зависит от структуры совместной функции распределения вероятности $w\left(m_{1}, m_{2}, \vec{n}_{1}, \vec{n}_{2}\right)$. Мы обсудим три случая.

СлУчАй 1. Совместная функция распределения вероятностей факторизуется,

$$
w\left(m_{1}, m_{2}, \vec{n}_{1}, \vec{n}_{2}\right)=w\left(m_{1}, \vec{n}_{1}\right) w\left(m_{2}, \vec{n}_{2}\right) .
$$

Это известное условие факторизации Белла-Клаузера-Хорне [21]. Оно обсуждается также в работе [22].

В этом случае коррелятор имеет вид

$$
\begin{gathered}
\left\langle M_{\alpha \beta}\right\rangle=w(1, \vec{\alpha}) w(1, \vec{\beta})-w(1, \vec{\alpha}) w(-1, \vec{\beta})-w(-1, \vec{\alpha}) w(1, \vec{\beta})+ \\
+w(-1, \vec{\alpha}) w(-1, \vec{\beta}), \quad \alpha=a, d, \quad \beta=b, c .
\end{gathered}
$$

При этом формула (34) может быть переписана как

$$
\begin{aligned}
\left\langle M_{a b}\right\rangle+ & \left\langle M_{a c}\right\rangle+\left\langle M_{d b}\right\rangle-\left\langle M_{d c}\right\rangle= \\
& =-w(1, \vec{a})[w(1, \vec{b})+w(1, \vec{c})]-w(1, \vec{a})[w(-1, \vec{c})+w(-1, \vec{c})]- \\
& -w(-1, \vec{a})[w(1, \vec{b})+w(1, \vec{c})]+w(-1, \vec{a})[w(-1, \vec{b})+w(-1, \vec{c})]+ \\
& +w(1, \vec{d})[w(1, \vec{b})-w(1, \vec{c})]-w(1, \vec{d})[w(-1, \vec{b})-w(-1, \vec{c})]- \\
& -w(-1, \vec{d})[w(1, \vec{b})-w(1, \vec{c})]+w(-1, \vec{d})[w(-1, \vec{b})-w(-1, \vec{c})] .
\end{aligned}
$$

Учитывая условие

$$
w(1, \vec{\gamma})+w(-1, \vec{\gamma})=1, \quad \gamma=a, b, c, d,
$$

получаем, что $\left|\left\langle M_{a b}\right\rangle+\left\langle M_{a c}\right\rangle+\left\langle M_{d b}\right\rangle-\left\langle M_{d c}\right\rangle\right| \leqslant 2$, и неравенство Белла в данном случае выполняется.

СлучАй 2. Совместная функция распределения вероятностей может быть представлена в виде суммы произведений функций распределения вероятностей переменных,

$$
w\left(m_{1}, m_{2}, \vec{n}_{1}, \vec{n}_{2}\right)=\sum_{k} p_{k} w^{(k)}\left(m_{1}, \vec{n}_{1}\right) W^{(k)}\left(m_{2}, \vec{n}_{2}\right),
$$

причем числа $p_{k}$ неотрицательны и удовлетворяют условию нормировки $\sum_{k} p_{k}=1$. Это условие рассматривалось Вигнером [23] и обсуждалось в недавней работе [24].

В этом случае мы получаем линейную комбинацию слагаемых, каждое из которых имеет вид, аналогичный (38), с неотрицательными коэффициентами. Каждое слагаемое меньше 2 , коэффициенты $p_{k}$ меньше единицы и удовлетворяют условию нормировки, следовательно, вся сумма также меньше 2, и неравенства Белла выполняются. 
СлучАй 3. Совместное распределение вероятностей имеет вид, отличный от предыдущих двух. В этом случае неравенства Белла могут нарушаться. В качестве примера рассмотрим совместное распределение вероятностей $w\left(m_{1}, m_{2}, \vec{n}_{1}, \vec{n}_{2}\right)$ следующего вида:

$$
\begin{aligned}
& w\left(1,-1, \vec{n}_{1}, \vec{n}_{2}\right)=w\left(-1,1, \vec{n}_{1}, \vec{n}_{2}\right)= \\
& =\frac{1}{2}\left(\cos ^{2} \frac{\theta_{1}}{2} \sin ^{2} \frac{\theta_{2}}{2}+\sin ^{2} \frac{\theta_{1}}{2} \cos ^{2} \frac{\theta_{2}}{2}\right)-\frac{1}{4} \sin \theta_{1} \sin \theta_{2} \cos \left(\psi_{1}+\psi_{2}\right), \\
& w\left(1,1, \vec{n}_{1}, \vec{n}_{2}\right)=w\left(-1,-1, \vec{n}_{1}, \vec{n}_{2}\right)= \\
& =\frac{1}{2}\left(\cos ^{2} \frac{\theta_{1}}{2} \cos ^{2} \frac{\theta_{2}}{2}+\sin ^{2} \frac{\theta_{1}}{2} \sin ^{2} \frac{\theta_{2}}{2}\right)+\frac{1}{4} \sin \theta_{1} \sin \theta_{2} \cos \left(\psi_{1}+\psi_{2}\right), \\
& \vec{n}_{2}=\left(\sin \theta_{2} \cos \phi_{2}, \sin \theta_{2} \sin \psi_{2}, \cos \theta_{2}\right) \text {. }
\end{aligned}
$$

Имеем

$$
\left\langle M_{\alpha \beta}\right\rangle=\cos \theta_{\alpha} \cos \theta_{\beta}+\sin \theta_{\alpha} \sin \theta_{\beta} \cos \left(\psi_{\alpha}+\psi_{\beta}\right), \quad \vec{\alpha}=\vec{a}, \vec{d}, \quad \vec{\beta}=\vec{b}, \vec{c} .
$$

Получаем, что

$$
\begin{aligned}
I= & \left\langle M_{a b}\right\rangle+\left\langle M_{a c}\right\rangle+\left\langle M_{d b}\right\rangle-\left\langle M_{d c}\right\rangle= \\
= & \cos \theta_{d}\left(\cos \theta_{b}+\cos \theta_{c}\right)+\sin \theta_{d}\left[\sin \theta_{b} \cos \left(\psi_{d}+\psi_{b}\right)+\sin \theta_{c} \cos \left(\psi_{d}+\psi_{c}\right)\right]+ \\
& \quad+\sin \theta_{a}\left[\sin \theta_{b} \cos \left(\psi_{a}+\psi_{b}\right)-\sin \theta_{c} \cos \left(\psi_{a}+\psi_{c}\right)\right]+\cos \theta_{a}\left(\cos \theta_{b}-\cos \theta_{c}\right) .
\end{aligned}
$$

Вычисляя $I$ для углов $\theta_{d}=\theta_{b}=\theta_{c}=\pi / 6, \theta_{a}=\pi / 2, \quad \psi_{d}+\psi_{b}=\psi_{a}+\psi_{c}=\pi / 4$, $\psi_{d}+\psi_{c}=-\pi / 2, \quad \psi_{a}+\psi_{b}=\pi$, получаем значение $I=5 / 4+5 \sqrt{2} / 8>2$. Мы видим, что в этом случае неравенства Белла нарушаются. Необходимо отметить, что рассмотренная в третьем случае спиновая томограмма задает перепутанные состояния Вернера для двух частиц со спинами $1 / 2$ с матрицей плотности

$$
\varrho=\frac{1}{2}\left(\begin{array}{llll}
1 & 0 & 0 & 1 \\
0 & 0 & 0 & 0 \\
0 & 0 & 0 & 0 \\
1 & 0 & 0 & 1
\end{array}\right)
$$

Переменные $m_{1}$ и $m_{2}$ в этом случае имеют физический смысл удвоенных проекций спина на направления, заданные векторами $\vec{n}_{1}$ и $\vec{n}_{2}$, соответственно.

Итак, мы получили нарушение неравенств Белла в случае перепутанных состояний. Необходимо отметить, что факт нарушения неравенств Белла для перепутанных квантовых состояний составных систем был известен и ранее, также как и возможность использования нарушения неравенств Белла в каких-либо состояниях в качестве критерия перепутанности этих состояний. В предложенном нами рассмотрении новым является использование при рассмотрении неравенств Белла 
вероятностного представления квантовой механики и обсуждение нарушений неравенств Белла для квантовых состояний составных систем, описываемых совместными функциями распределения вероятностей (томограммами состояний), с использованием стандартных в классической теории вероятности правил вычисления моментов в применении к квантовым переменным.

Рассмотрим теперь для состояния Вернера (27) квантовомеханическое неравенство Белла, построенное в работах [25].

Если спиновое двухчастичное состояние описывается матрицей плотности

$$
\rho=\left\|\rho_{i j}\right\|, \quad i, j=1,2,3,4,
$$

то значение спиновой корреляции можно представить в виде

$$
E(\vec{a}, \vec{b})=\operatorname{Tr}(\hat{a} \otimes \hat{b} \rho)=\left(a_{x} a_{y} a_{z}\right) P\left(\begin{array}{l}
b_{x} \\
b_{y} \\
b_{z}
\end{array}\right)=(\vec{a}, P \vec{b}),
$$

где $P$ - матрица размера $3 \times 3$,

$$
P=\left(\begin{array}{ccc}
\left(\rho_{14}+\rho_{23}+\rho_{32}+\rho_{41}\right) & i\left(\rho_{14}-\rho_{23}+\rho_{32}-\rho_{41}\right) & \left(\rho_{13}+\rho_{31}-\rho_{24}-\rho_{42}\right) \\
i\left(\rho_{14}+\rho_{23}-\rho_{32}-\rho_{41}\right) & \left(-\rho_{14}+\rho_{23}+\rho_{32}-\rho_{41}\right) & i\left(\rho_{13}-\rho_{31}-\rho_{24}+\rho_{42}\right) \\
\left(\rho_{12}+\rho_{21}-\rho_{34}-\rho_{43}\right) & i\left(\rho_{12}-\rho_{21}-\rho_{34}+\rho_{43}\right) & \left(\rho_{11}-\rho_{22}-\rho_{33}+\rho_{44}\right)
\end{array}\right) .
$$

Для состояния (40) выполняется квантовомеханическое неравенство Белла

$$
|E(\vec{a}, \vec{b})+E(\vec{a}, \vec{c})+E(\vec{d}, \vec{b})-E(\vec{d}, \vec{c})| \leqslant \sqrt{2} \sup _{n_{1}, n_{2}}\left(\left\|P\left(n_{1}\right)\right\|+\left\|P\left(n_{2}\right)\right\|\right),
$$

где точная верхняя грань вычисляется по всем парам векторов $n_{1}, n_{2}$ таким, что

$$
\left(n_{1}, n_{2}\right)=0, \quad\left\|n_{1}\right\|=\left\|n_{2}\right\|=1 .
$$

Для состояния Вернера (27) матрица (42) имеет вид

$$
P=\left(\begin{array}{ccc}
p & 0 & 0 \\
0 & -p & 0 \\
0 & 0 & p
\end{array}\right)
$$

Таким образом, в этом случае выполняется квантовомеханическое неравенство Белла

$$
|E(\vec{a}, \vec{b})+E(\vec{a}, \vec{c})+E(\vec{d}, \vec{b})-E(\vec{d}, \vec{c})| \leqslant 2 p \sqrt{2}
$$

Как уже отмечалось, в соответствии с критерием Переса-Городецкого, если параметр $p$ удовлетворяет неравенству $-1 / 3 \leqslant p \leqslant 1 / 3$, то состояния Вернера (27) являются различимыми (неперепутанными). Если же $1 / 3<p<1$, то состояние Вернера будет перепутанным. Из соотношения (44) следует, что если $1 / 3<p<\sqrt{2} / 2$, то для таких перепутанных состояний неравенство Белла (35) не будет нарушаться. Можно вычислить совместное распределение двух проекций спина на свои направления для состояния Вернера (27), зависящего от параметра $p$. Расчет максимума величины, стоящей в левой части неравенства (44), проведенный с помощью этой совместной функции распределения вероятностей, приводит к тому же результату. 


\section{5. ЗАКЛЮЧЕНИЕ}

Мы показали, что спиновые состояния могут описываться стандартными функциями распределения вероятностей вместо спиноров или матриц плотностей как в случае одной частицы со спином, так и в случае нескольких частиц со спинами. Спиновая томография, представляющая собой вероятностное представление для спиновых состояний, является специфическим вариантом звездочного квантования. Кроме того, мы предложили в рамках вероятностного представления спиновых состояний рассмотреть проблему перепутанности квантовых состояний системы двух частиц со спинами с точки зрения нарушения или выполнения неравенств Белла, подчеркнув зависимость этого нарушения от структуры совместной функции распределения вероятности.

Мы показали, что неравенства Белла гарантированно выполняются для совместных функций распределения вероятностей, обладающих структурой, эквивалентной структуре таких функций, которые соответствуют в квантовой механике сепарабельным (разделимым) состояниям. Мы продемонстрировали, что в классической теории вероятностей существуют совместные функции распределения, не имеющие такой структуры и приводящие к нарушению неравенств Белла. Неравенство Белла - это свойство совместной функции распределения вероятностей, проявляющееся в виде свойства вторых моментов (корреляторов) таких функций. Из проведенного анализа очевидно, что упомянутые различия в структуре совместных функций распределения вероятностей могут проявляться и в свойствах других высших моментов этих функций распределения.

Благодарности. Данное исследование было поддержано РФФИ (грант № 0302-16408).

\section{Список литературы}

[1] J. S. Bell. Physics. 1964. V. 1. P. 195.

[2] S. Mancini, V. I. Man'ko, P. Tombesi. Phys. Lett. A. 1996. V. 213. P. 1; Found. Phys. 1997. V. 27. P. 801.

[3] V. V. Dodonov, V. I. Man'ko. Phys. Lett. 1997. V. 239. Р. 335; В. И. Манъко, О. В. Манько. ЖЭТФ. 1997. Т. 112. С. 796.

[4] В. И. Манъко, С. С. Сафонов. ТМФ. 1997. Т. 112. № 3. С. 467; ЯФ. 1998. Т. 61. C. 658 .

[5] В. А. Андреев, В. И. Манъко. ЖЭЭТФ. 1998. Т. 114. С. 437; V. A. Andreev, O. V. Man'ko, V. I. Man'ko, S. S. Safonov. J. Russ. Laser Res. 1998. V. 19. P. 340.

[6] S. Mancini, O. V. Man'ko, V. I. Man'ko, P. Tombesi. J. Phys. A. 2001. V. 34. P. 3461.

[7] O. V. Man'ko, V. I. Man'ko, G. Marmo. Phys. Scripta. 2000. V. 62. P. 446; J. Phys. A. 2002. V. 35. P. 699.

[8] J. E. Moyal. Proc. Cambridge Philos. Soc. 1949. V. 45. P. 99.

[9] I. Fenyes. Z. Phys. 1952. V. 132. Р. 81; С. Л. Стратонович. ЖЭТФ. 1956. Т. 31. C. 1012; F. Bayen, M. Flato, C. Fronsdal, A. Lechnerovicz, D. Sternheimer. Lett. Math. Phys. 1975. V. 1. Р. 521; И. В. Тютин. ТМФ. 2001. Т. 127. № 2. С. 253; M. A. Vasiliev. Fortschr. Phys. 1988. V. 36. P. 33; B. Fedosov. J. Differential Geom. 1994. V. 40. P. 213; M. Kontsevich. Lett. Math. Phys. 2003. V. 66. P. 157; q-alg/9709040. 
[10] G. G. Amosov, V. I. Man'ko. Phys. Lett. A. 2003. V. 318. P. 287; J. Russ. Laser. Res. 2004. V. 25. P. 253.

[11] В. А. Андреев, В. И. Манъко. ТМФ. 2004. Т. 140. № 2. С. 284.

[12] O. V. Man'ko, V. I. Man'ko. J. Russ. Laser Res. 2004. V. 25. P. 115; quant-ph/041131.

[13] A. С. Холево. Введение в квантовую теорию информации. М.: МЦНМО, 2002; А. Ю. Хренников. Неколмогоровские теории вероятностей и квантовая физика. М.: Физматлит, 2003.

[14] A. Khrennikov. Found. Phys. 2002. V. 32. P. 1159; A. Khrennikov, I. Volovich. Local Realism, Contextualism and Loopholes in Bell's Experiments. quant-ph/0212127; Quantum nonlocality, EPR model and Bell's theorem. In: Proc. 3rd International Sakharov Conference on Physics. V. 2 (Moscow, June 24-29, 2002). Eds. A. Semikhatov, M. Vasiliev, V. Zaikin. Singapore: World Scientific, 2003. P. 260-267.

[15] I. V. Volovich. Quantum information in space and time and theory of stochastic processes. In: Quantum information. IV. Proc. 4-th Int. Conf. (Nagoya, Japan, February 27 - March 1, 2001). Eds. T. Hida, K. Saito. Singapore: World Scientific, 2002. P. 187-200; Quantum cryptography in space and Bell's theorem. In: Foundation of Probability and Physics. PQ-QP: Quantum Probabilility and White Noise Analysis. V. 13. Proc. Conf. (Vaxjo, Sweden, November 27 - December 1, 2000). Ed. A. Khrennikov. Singapore: World Scientific, 2001. P. 364-372.

[16] V. A. Andreev, V. I. Man'ko. The Quantum Tomography Representation for Bell-CHSH inequalities. In: Proc. Int. Conf. Quantum Theory: Reconsideration of Foundations-2 (Vaxjo, Sweden, June 1-7, 2003). Ed. A. Khrennikov. Vaxjo: Vaxjo Univ. Press, 2004. P. $47-54$.

[17] V. A. Andreev, V. I. Man'ko. J. Opt. B. Quantum Semiclass. Opt. 2000. V. 2. P. 122.

[18] A. B. Klimov, O. V. Man'ko, V. I. Man'ko, Yu. F. Smirnov, V. N. Tolstoy. J. Phys. A. 2002. V. 35. P. 6101.

[19] V. I. Man'ko, G. Marmo, E. C. G. Sudarshan. Phys. Lett. A. 2004. V. 327. P. 353.

[20] A. Peres. Phys. Rev. Lett. 1996. V. 77. P. 1413; M. Horodecki, P. Horodecki, R. Horodecki. Phys. Lett. A. 1996. V. 223. P. 1; quant-ph/9605038.

[21] A. Shimony. Search for a Naturalistic World View. Cambridge, MA: Cambridge Univ. Press, 1993.

[22] A. Khrennikov. Comment on Hess-Philipp anti-Bell and Gill-Weihs-Zeilinger-Zukowski anti-Hess-Philipp arguments. quant-ph/0205022 v1.

[23] E. Wigner. Quantum-mechanical distribution functions revisted. In: Perspectives in Quantum Theory. Eds. W. Yourgrau, van der A. Merwe. Cambridge, MA: MIT Press, 1971.

[24] A. Khrennikov. Phys. Essays. 2003. V. 16. № 2. P. 26.

[25] В. А. Андреев, В. И. Манъко. Письма в ЖЭТФ. 2000. Т. 72. С. 130; V. A. Andreev, V. I. Man'ko. Phys. Lett. A. 2001. V. 281. P. 278. 